\title{
Human mesenchymal stem cells derived from amniotic membrane attenuate isoproterenol (ISO)-induced myocardial injury by targeting apoptosis
}

\author{
Maryam Kheila ${ }^{1 \gamma}$, Fazel Gorjipour ${ }^{2 \gamma}$, Ladan Hosseini Gohari ${ }^{2}$, Masoomeh Sharifi $^{1}$, Nahid Aboutaleb ${ }^{1^{*}}$ (D) \\ Received: 11 Apr 2019 \\ Published: 26 Jun 2021
}

\section{Abstract}

Background: Currently, stem cell therapy has been proposed as an efficient strategy to prevent or treat myocardial injuries. The current study was conducted to examine cardioprotective effects of human mesenchymal stem cells derived from amniotic membrane (hAMSCs) against isoproterenol (ISO)-induced myocardial injury and explore its potential mechanisms.

Methods: The hAMSCs were injected intramyocardially in male Wistar rats 28 days after last injection of ISO (170 mg/kg body weight for 4 consecutive days). The echocardiography was performed to confirm induction of myocardial damage and cardiac function 28 days after last injection of ISO and 4 weeks hAMSCs transplantation after HF induction. The expression of apoptotic markers such as Bcl-2, Bax and P53 was evaluated using Western blotting assay. Masson's trichrome staining was used to determine fibrosis. The cytoarchitecture of myocardial wall and morphology of cells were investigated using hematoxylin and eosin (H\&E) staining.

Results: As compared to ISO group, hAMSCs transplantation after heart failure (HF) induction significantly blunted the increasing of cardiac dimensions and restored ejection fraction (EF) and fractional shortening (FS) parameters $(\mathrm{p}<0.05)$. Moreover, hAMSCs transplantation after HF induction increased the expression of antiapoptotic markers such as Bcl-2 and decreased the expression of proapoptotic markers such as P53 and Bax $(\mathrm{p}<0.05)$. As compared to ISO group, hAMSCs transplantation after HF induction markedly reduced interstitial myocardial fibrosis and contributed to maintain of normal cytoarchitecture of myocardial wall and morphology of cells.

Conclusion: Collectively, the results of current study suggest that transplantation of hAMSCs confers cardioprotection by targeting ISO-induced mitochondria-dependent (intrinsic) pathway of apoptosis.

Keywords: Cardiac injury, Apoptosis, Human mesenchymal stem cells, Cardiac function, Fibrosis

Conflicts of Interest: None declared

Funding: Dean of Research, Iran University of Medical Sciences

\section{*This work has been published under CC BY-NC-SA 1.0 license.} Copyright $\odot$ Iran University of Medical Sciences

Cite this article as: Kheila M, Gorjipour F, Hosseini Gohari L, Sharifi M, Aboutaleb N. Human mesenchymal stem cells derived from amniotic membrane attenuate isoproterenol (ISO)-induced myocardial injury by targeting apoptosis. Med J Islam Repub Iran. 2021 (26 Jun);35:82. https://doi.org/10.47176/mjiri.35.82

\section{Introduction}

Despite new developments introduced into the health area and increasing the awareness about the personal and public health, cardiovascular disorders are among the leading causes of death around the world $(1,2)$. Cardiovascular disorders can lead to irreversible changes such as

Corresponding author: Dr Nahid Aboutaleb, aboutaleb.n@iums.ac.ir

${ }^{\gamma}$ These authors have equally contributed to this research work.

1. Physiology Research Center, Iran University of Medical Sciences. Tehran, Iran

2. Cellular and Molecular Research Center, Iran University of Medical Sciences. Tehran, Iran hypertrophy, dilatati on, initiation of acute inflammatory responses, the necrosis of the myocytes and subsequently collagen scar formation $(3,4)$. One of the common pathologic consequences of myocardial injury is fibrosis and is characterized by abnormal accumulation of the extracellu-

$\uparrow$ What is "already known" in this topic:

For improving heart function in ISO-induced myocardial injury administration of hAMSCs can be useful.

$\rightarrow$ What this article adds:

ISO-induced myocardial injury has been related with cardiac dysfunction and apoptotic cell death. Since MSCs transplantation after HF prevents apoptotic cell death, it improves outcomes in HF model through antiapoptotic effects. 
lar matrix (ECM) proteins rich in fibrillar collagens which, in turn, leads to the progression of cardiovascular diseases towards heart failure (HF) (5). Other pivotal factor for the cardiac injury is oxidative stress and is caused by increased level of reactive oxygen species (ROS) in the myocytes (6). Indeed, increased level of ROS gives rise to the oxidative damage of proteins, lipids, membranes, and DNA in subcellular organelles and tissue which, in turn, result in apoptotic cell death (7). Bcl-2 family proteins are responsible to control apoptotic mitochondrial pathway through regulation of mitochondrial membrane permeability. The $\mathrm{p} 53$ is a master regulator of the Bcl-2 family proteins that contributes to activation of caspases and subsequently rapid cell death (8). A short-term therapy and fundamental intervention in the insufficient circulation in acutely ill patients is injection of exogenous catecholamines that can induce sustained adrenergic stress and subsequently cardiomyopathy. Isoproterenol (ISO) is a synthetic catecholamine that acts as $\beta$-adrenergic agonist and creates intense stress and consequently infarct-like necrosis in the cardiac tissue (9). The quinone compounds can be formed via the oxidation of catecholamine and generate superoxide anions and subsequently hydrogen peroxide. Likewise, in the presence of iron, hydrogen peroxide creates devastating hydroxyl radicals that, in turn, enhance necrotic and apoptotic cell death. In recent years, researchers have used many new therapeutic agents and method to treat degenerative disorders (10-12). Owing to unique properties such as, differentiation plasticity, facile attainability, suppression of inflammation and low immunogenicity, mesenchymal stem cells (MSCs) have gained the attention of scientific community for treatment of myocardial injury $(13,14)$. For example, Zhang et al. investigated the ability of transplanted MSCs for amelioration of cardiac fibrosis following isoproterenol-induced HF (15). They found that transplanted MSCs could ameliorate myocardial fibrosis due to their paracrine signaling and secretion of antifibrotic factors (15). Moreover, it has been reported that MSCs transplantation after HF induction in rats resulted in decreased level of apoptotic markers such as P53, oxidative stress markers such as nuclear-related factor-2 (Nrf2) and inflammatory markers such as toll-like receptor-4 (TLR-4) (16). Here, we evaluated the cardioprotective effects of human mesenchymal stem cells derived from amniotic membrane (hAMSCs) on a rat model of ISO-induced myocardial damage. Present study examines the effects of hAMSCs on apoptosis pathway following ISO-induced myocardial damage.

\section{Methods}

This is an experimental study in which, the effect of hAMSCs on ISO-induced myocardial injury was examined by targeting apoptosis.

\section{Reagents}

Anti-Bax antibody (ab53154), Anti-Bcl-2 antibody (ab59348), and Anti-p53 antibody (ab131442) were purchased from Abcam Company (Cambridge, UK). Immobilon ${ }^{\circledR}-\mathrm{FL}$ Polyvinylidene difluoride (PVDF) Membrane, Bovine serum albumin (BSA), and RIPA buffer were sup- plied from Sigma Company (St. Louis, MO, USA). Human amniotic membrane-derived mesenchymal cells were obtained from Iranian Blood Transfusion Organization.

\section{Animals and ethical statement}

Male Wistar rats weighing 180-200 grams were procured from Animal Center of Iran University of Medical Sciences, Tehran, Iran. The rats were acclimatized in a controlled room temperature $\left(22 \pm 2{ }^{\circ} \mathrm{C}\right)$ with humidity about $62-65 \%$ under a 12 -h light and dark schedule one week before the experiments. They fed with standard pellet diet and water available. Experimental protocols were approved by the institutional ethics review board at Iran University of Medical Sciences (IUMS, Tehran, Iran; ethics committee approval code: IR.IUMS.REC.1393.25477).

\section{Induction of myocardial injury}

To induce myocardial injury, ISO $(170 \mathrm{mg} / \mathrm{kg}$ body weight, dissolved in saline) was subcutaneously injected once a day for 4 consecutive days.

\section{Experimental design}

The animals were allocated into three groups:

Group1: This group includes healthy male Wistar rats receiving sham operation (Control).

Group 2: In this group, the animals subcutaneously received ISO $(170 \mathrm{mg} / \mathrm{kg}$ body weight, dissolved in saline for 4 consecutive days and sham operation (ISO).

Group 3: In this group, rats were subjected to cardiac injury through ISO injection (170 mg/kg body weight) and intramyocardially received hAMSCs (ISO+ hAMSCs). HAMSCs were prepared and injected as previously described (17). Both sham operation and hAMSCs injection were performed in the same way to control for stress induced by chest opening and intramyocardial injection. Sham operation included chest opening and phosphatebuffered saline (PBS) injection. PBS was used as the carrier for cells

\section{Cardiac function analysis}

A $10-\mathrm{MHz}$ probe interfaced with a GE Vivid Cardiovascular high-frequency ultrasound system (GE Healthcare, Chicago, Illinois, United States) was used to perform echocardiography. Baseline echocardiography was carried out to ensure the animals were normal prior to starting of experiments.

To confirm myocardial damage, echocardiography was performed 28 days after the final ISO injection. Another echocardiography was carried out 4 weeks after hAMSCs transplantation. Briefly, rats were anesthetized using a mixture of ketamine $(50 \mathrm{mg} / \mathrm{kg})$ and xylazine $(5 \mathrm{mg} / \mathrm{kg})$, and their chest was shaved. A warming pad was used to modulate normothermia. The animals were placed in the left lateral position, acoustic coupling gel was scrubbed and ECG (Electrocardiogram) was obtained. The parasternal left ventricular short axis view at the level of papillary muscles was used to determine the left ventricular endsystolic internal dimension (LVIDs) and left ventricular end-diastolic internal dimension (LVIDd) in MMode. Finally, calculation of ejection fraction (EF) and fractional 
shortening (FS) parameters were performed based on the following formula: $\mathrm{EF}=\left(\mathrm{LVIDd}^{2}-\mathrm{LVIDs}^{2}\right) / \mathrm{LVIDd}^{2}$, $\mathrm{FS}=((\mathrm{LVIDd}-\mathrm{LVIDs}) / \mathrm{LVIDd}) \times 100$.

\section{Western blotting analysis}

First, the frozen tissues were recovered from $-80^{\circ} \mathrm{C}$ storage and then lysed using RIPA lysis buffer containing complete protease inhibitor cocktail. Heart lysates were centrifuged at $16,400 \mathrm{rpm}$ for 20 minutes. Then, a nanodrop instrument was used to determine protein concentrations. In next step, 4-20\% gradient SDS/PAGE (sodium dodecyl sulfate polyacrylamide gel electrophoresis) was used to separate $30 \mu \mathrm{g}$ soluble protein and then transferred to PVDF membranes. Then, PVDF membranes were incubated in 5\% nonfat milk (diluted in Trisbuffered saline (TBS)) for one hour to block nonspecific binding. In next step, PVDF membranes were incubated overnight at $4{ }^{\circ} \mathrm{C}$ with primary antibodies including AntiBax antibody, Anti-Bcl-2 antibody, and Anti-p53 antibody. Membranes were washed with TBS with Tween-20 (TBST) for several times and then were exposed to the secondary antibodies diluted in blocking buffer for one hour at $37{ }^{\circ} \mathrm{C}$. Finally, chemilumiescence reagents (Thermo scientific, Massachusetts, United States) ahead of XOMAT films (The Eastman Kodak, New York, USA) were used to determine immunoreactivity, all according to the suppliers notes. The protein levels were normalized to those of $\beta$-actin and were quantified using the Image $J$ software (NIH, Bethesda, MD, USA).

\section{Apoptosis assays}

To evaluate apoptosis and DNA fragmentation in cardiac tissue, terminal deoxynucleotidyl transferase dUTPend-labeling (TUNEL) staining was performed using an In Situ Cell Death Detection Kit (Roche Molecular Biochemicals, Germany). In brief, the heart tissues were fixed in formalin and embedded in paraffin. Using Microtome, 5 $\mu \mathrm{m}$ thick sections from base to apex were prepared. The sections were washed with PBS for several times and then were incubated with $10 \mathrm{mM}$ proteinase $\mathrm{K}$ at $25^{\circ} \mathrm{C}$ for 30 min to permeabilize them. In next step, the sections were incubated with $3 \% \mathrm{H}_{2} \mathrm{O}_{2}$ in methanol for $15 \mathrm{~min}$ to inhibit endogenous peroxidase activity and subsequently prevent false positive results. Once again, the sections were washed with cold PBS and then treated with TUNEL reaction buffer for 15 minutes and then were incubated with the TUNEL reaction mixture (containing label solution and enzyme) for one hour in a moderate humidified chamber with a temperature about $37-40^{\circ} \mathrm{C}$. Then, the sections were labeled with anti-fluorescein antibodyconjugated with horseradish peroxidase for 15 minutes. Treatment with $0.05 \%$ DAB (3, 3-diaminobanzidine) substrate as a chromogen was performed for 2 min to provide possibility for visualization of the apoptotic cells. Likewise, in order to stain the cells background, the Gill hematoxylin was applied. In the case of each experimental group, four animals were used to prepare sections. For each section, five random fields were chosen and 100 cells were counted per field by an examiner blinded to experimental design. Counting was carried out using a light mi- croscope (Olympus, Hamburg, Germany).

\section{Histological assessment}

Interstitial myocardial fibrosis was determined using Masson's trichrome staining. Briefly, the heart tissues were fixed in formalin and embedded in paraffin. Using Microtome, $5 \mu \mathrm{m}$ thick sections from base to apex were prepared. The Image J software (NIH, Bethesda, MD, USA) was applied to quantify' data via color deconvolution plugin. In the case of Masson's trichrome staining, blue parts in images show collagen synthesis and deposition. In the case of each experimental group, four animals were used to prepare sections and 10 sections were prepared.

Tissue damage was evaluated using hematoxylin and eosin (H\&E) method. In the case of $\mathrm{H} \& \mathrm{E}$, the cytoarchitecture of myocardial wall, morphology, and nuclear condensation were evaluated by an expert blinded to experimental design.

\section{Statistical analysis}

Data were presented as means \pm standard deviations (SD). Data analysis was performed using GraphPad PRISM5 (Graphpad Inc., La Jolla, USA). To compare variations among two groups, two-tailed Student's t-test was used. In order to compare variations among experimental groups, one-way analysis of variance followed by Tukey test for post hoc analysis was used.

\section{Results}

\section{Cardiac function}

We evaluated whether myocardial injury was induced 28 days after the final ISO injection and transplantation of hAMSCs affect cardiac function. The results of echocardiography demonstrated that LVIDd and LVIDs were significantly increased in HF group compared to control which, in turn, resulted in significant decreases in EF and FS (Fig. 1 A and B). Transplantation of hAMSCs markedly blunted increasing of LVIDd and LVIDs and subsequently restored EF and FS compared to HF group (Fig. 1 C and D).

\section{Cardiac fibrosis}

To further explore the cardiac protective effect of hAMSCs, we carried out Masson's trichrome staining. As shown in Figure 2, a significant increased interstitial myocardial fibrosis was observed in HF group compared to control. Transplantation of hAMSCs markedly attenuated ISO-triggered fibrosis after 4 weeks.

\section{The expression of the apoptiotic markers}

Western blotting assay was furtherly used to investigate the expression of the apoptotic markers such as p53, Bcl-2 and Bax. Our results demonistrated that ISO treatment significantly increased the expression of $\mathrm{p} 53$ and Bax proteins (Fig. 3 A and B). Significant reduced levels of p53 and Bax proteins were observed in the treatment group. Following, western blotting analysis demonstrated that the expression of Bcl-2 was highly decreased in ISO group. 
A

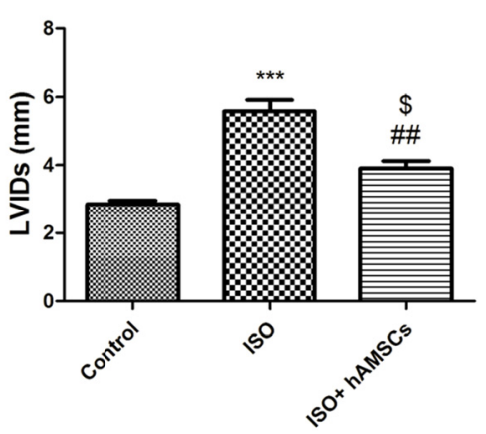

C

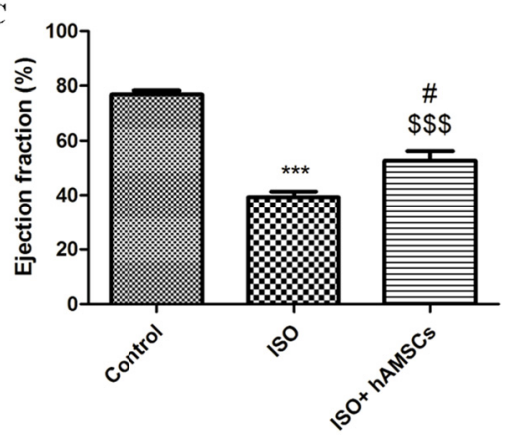

B

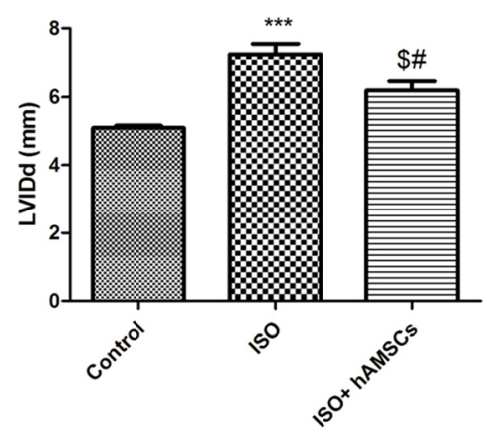

D

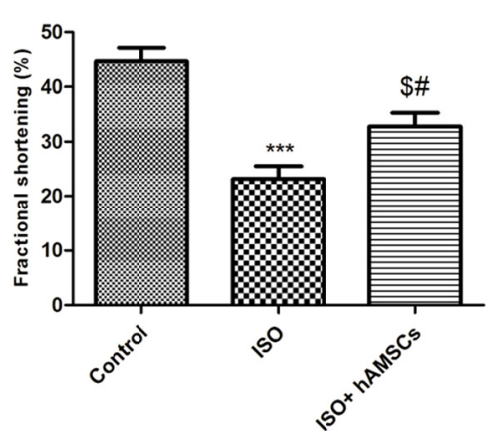

Fig. 1. The effects of MSCs transplantation after HF induction on heart function parameters in rats. A, B) ISO treatment significantly increased LVIDd and LVIDs that was reversed by MSCs transplantation after HF induction. C, D) The MSCs transplantation after HF induction markedly restored EF and FS parameters. MSC: mesenchymal stem cell, LVIDd: Left ventricular diameter in diastole, LVIDs: Left ventricular diameter in systole, EF: Ejection fraction, and FS: Fractional shortening (***P<0.001vs. Control; \#P<0. 05 and \#\#P<0. 01 vs. ISO; $\$ \mathrm{P}<0.05$ and $\$ \$ \$$ $\mathrm{P}<0.001$ vs. Control)

A
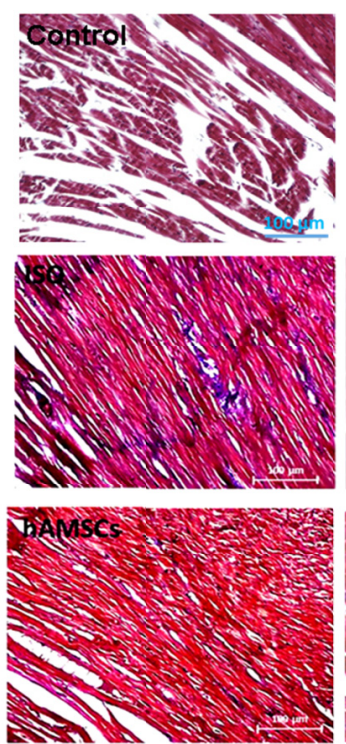

B
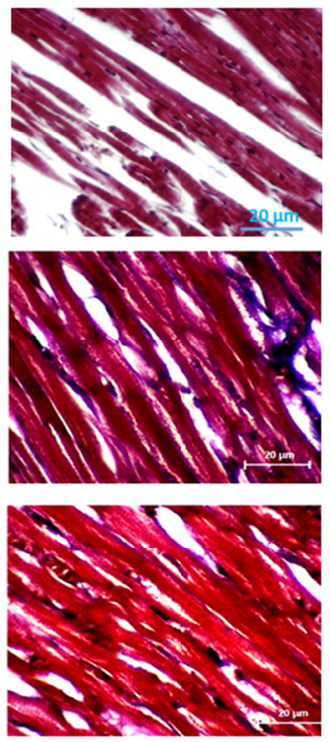

Fig. 2. The effects of MSCs transplantation after HF induction on collagen deposition in myocardium tissue of rats. A) Low (left, 100um scale bar) and high (right, 20um scale bar) magnification images of heart tissues for ISO and ISO+hAMSCs. B) Quantitative analysis of collagen deposition in myocardium tissue .ISO isoproterenol, hAMSCs human mesenchymal stem cells derived from amniotic membrane $(* * * \mathrm{P}<0.001 v s$. Control; \#P<0. 05 vs.ISO; $\$ \mathrm{P}<0.05$ vs. Control).

Transplantation of hAMSCs significantly elevated Bcl-2 after 4 weeks (Fig. 3 C).

In order to confirm anti-apoptotic effects of hAMSCs on ISO-induced myocardial damage, TUNEL staining was performed. TUNEL analysis showed that ISO treatment markedly increased the number of TUNEL positive cardiomyocytes (Fig. 4). This alteration was markedly ameliorated following hAMSC transplantation (4 weeks). 
A

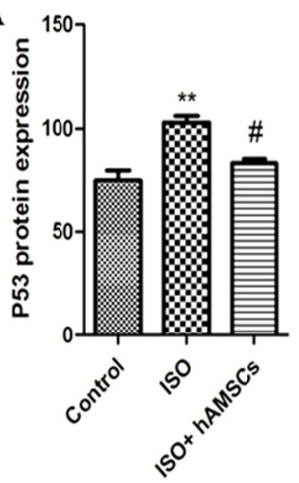

P53

$\beta$-actin
B

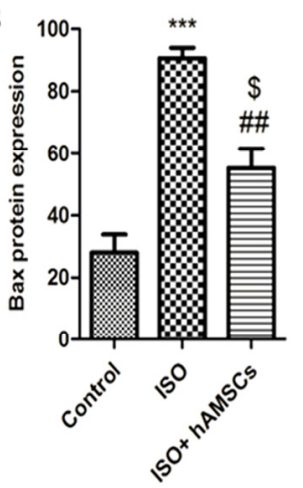

Bax

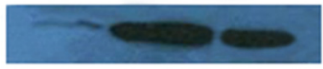

$\beta$-actin

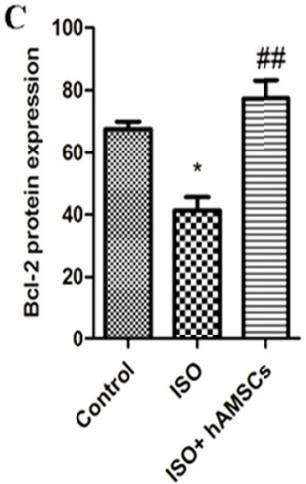

BCL2

$\beta$-actin

Fig. 3. The effects of MSCs transplantation after HF induction on the expression of pro-apoptotic markers and the expression of anti-apoptotic markers. A, B) a significant elevated level of p53 and Bax proteins were observed in ISO group. The MSCs transiplantation after HF induction reduced the levels of these proteins C) ISO treatment markedly decreased the expression level of Bcl-2 protein. An increased expression level of Bcl-2 protein was found following the post-MSC transplantation. ISO: isoproterenol, hAMSCs: human mesenchymal stem cells derived from amniotic membrane $(* \mathrm{P}<0.05, * * \mathrm{P}<0.01$ and $* * * \mathrm{P}<0.001$ vs. Control; \#\#P<0. 01 and $\# \mathrm{P}<0.05$ vs.ISO; $\$ \mathrm{P}<0.05$ vs Control).

A
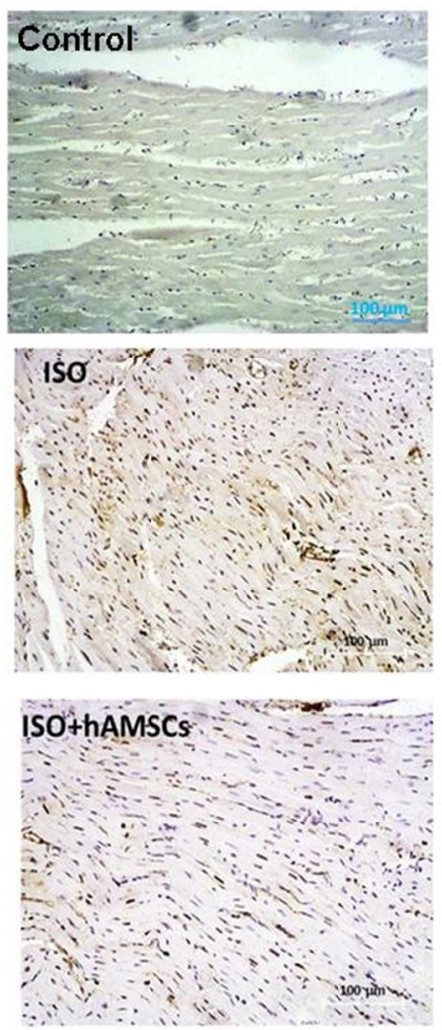

B
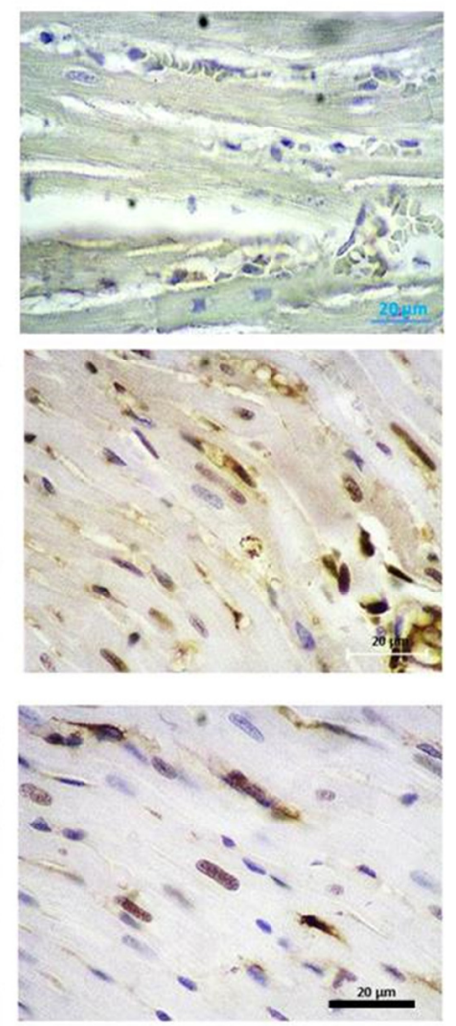

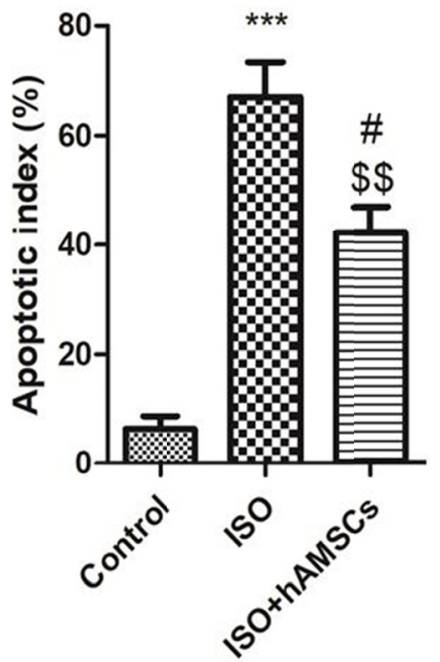

Fig. 4. The effects of MSCs transplantation after HF induction on the number of TUNEL positive cardiomyocytes in rats. A) Low (left, 100um scale bar) and high (right, 20um scale bar) magnification images of heart tissues for ISO and ISO+hAMSCs. B) Quantitative analysis of apoptotic cell death. ISO: isoproterenol, hAMSCs: human mesenchymal stem cells derived from amniotic membrane ( $* * * \mathrm{P}<0.001 \mathrm{vs}$. Control; \#P<0.05 vs.ISO; $\$ \$ \mathrm{P}<0.01$ vs. Control).

The effects of hAMSC transplantation on morphology of cardiomyocytes

As depicted in Figure 5, an abnormal cytoarchitecture of the myocardial wall and chromatin condensation were found in ISO group. Transplantation of hAMSCs improved cytoarchitecture of the myocardial wall and attenuate level of chromatin condensation compared to ISO group. 

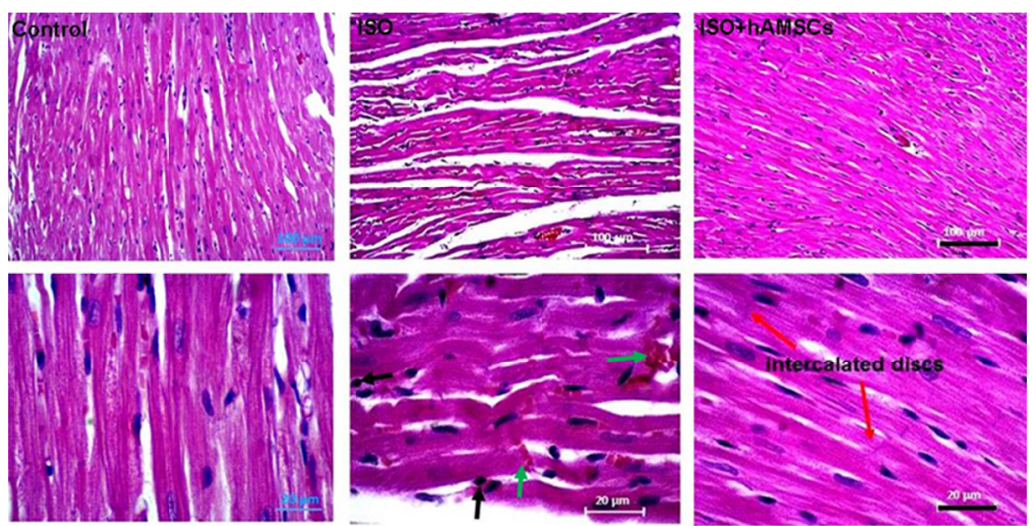

Fig. 5. The effects of MSCs transplantation after HF induction on cytoarchitecture of the myocardial wall and chromatin condensation. Low (left, 100um scale bar) and high (right, 20um scale bar) magnification images of heart tissues for ISO and ISO+hAMSCs .Black arrows show nuclear condensation while green arrows demonstrate abnormality of cytoarchitecture of the myocardial wall.

\section{Discussion}

In this study, we demonstrated that transplantation of hAMSCs ameliorated ISO-induced development of cardiac injury in male Wistar rats. We also attempted to show the possible molecular mechanisms of this cell therapy. The beneficial effect of hAMSCs seems to be linked to inhibition of apoptosis. Previous studies have shown that sub maximal doses of ISO can lead to blunted response to $\beta$-AR stimulation in myocardium, high expression of inducible nitric oxide synthase (iNOS), excessive production of nitric oxide (NO) and its by-products such as 3NTYR , activation of apoptotic signaling pathway and consequently heart dysfunction (18). In agreement with previous studies, our findings indicated that ISO treatment led to significant increases in dimensions of Left ventricular at end systole and diastole. Alteration of these functional parameters resulted in a significant reduction of EF and FS that were reversed by transplantation of hAMSCs. Previous studies have proposed many molecular mechanisms following administration of isoproterenol including massive inflammatory cell infiltration, release of proinflammatory cytokines, activation of xanthine oxidase (XO), and excessive production of ROS (19). Generally, initiation of acute inflammatory responses gives rise to ECM deposition in myocardium and subsequently the onset and progression of fibrosis (20). The mechanical and electrical functions of heart can be disrupted by cardiac fibrosis. In keeping with previous reports, our finding confirmed significant interstitial myocardial fibrosis formation following ISO treatment. Intramyocardial injection of hAMSCs reduced interstitial myocardial fibrosis that can be related to their paracrine effects according to previous studies (21). During pathological conditions such as myocardial ischemia reperfusion, chemical or druginduced ISO-induced myocardial damage like ISOinduced myocardial injury, impairment of the mitochondrial electron transport chain (ETC) gives rise to generation of ROS and reactive nitrogen species in large amounts, ATP depletion, overloading of intracellular $\mathrm{Ca}^{2+}$, mitochondrial swelling, structural changes of myocytes and finally apoptotic or necrotic cell death (22). Some previous studies have shown that ISO decreases the activities of mitochondrial respiratory chain enzymes such as cytochrome c oxidase and NADH dehydrogenase (23). In fact, ISO enhances intracellular accumulation of $\mathrm{Ca}^{2+}$ ion that, in turn, hampers the proton gradient within the mitochondrial membrane and reduces adenosine triphosphate (ATP) production (24). These alterations of mitochondrial membrane conduct cells towards apoptotic death. Apoptosis is a type of cell death modulated by a sequence of signal cascades during various conditions (25). Bcl-2 family proteins including $\mathrm{Bcl}-2$, Bcl- $\mathrm{Xl}$, and $\mathrm{Bcl}-\mathrm{W}$ are a group of fundamental factors that act as inhibitors of apoptotic cell death. On the other hand, Bax family including Bax, Bad, Bcl-xS and, Bak give rise to enhancement of apoptotic cell death (24). Previous studies have shown that ISO lead to significant changes in the expression of Bax/Bcl-2 family proteins. Overproduction of ROS by ISO triggers a sequential chain including activation of Bax, permeabilization of the external mitochondrial membrane and subsequently the release of mitochondrial cytochrome c. cytochrome $\mathrm{c}$ increases the activities of apoptotic proteaseactivating factor-1 (Apaf-1) and caspase-9, apoptosome formation and caspase-3 activation (26). On the other hand, P53 is a well-known transcription factor and a proapoptotic protein that mediate apoptosis through transactivating the expression of pro-apoptotic genes such as bax, caspase-6and, Apaf-1 (27). In this study, we found downregulation of antiapoptotic protein Bcl-2 and upregulation of Bax and P53 following ISO treatment. We observed transplantation of hAMSCs inhibited ISO-induced apoptosis by increased expression of Bcl-2 and decreased expression of P53 and Bax relative to ISO group. In agreement with our findings, a recent study by Kadry et al. showed that stem cell therapy following ISO -induced myocardial infarction contribute to improvement of heart function by targeting apoptotic marker P53 and Toll-Like Receptor-4/Nrf2 signaling pathway (16).in this study, we also confirmed inhibition of apoptosis using TUNEL staining. Our finding showed that transplantation of hAMSCs reduced the number of TUNEL-positive cells following ISO treatment. 


\section{Conclusion}

Collectively, this study demonstrates that transplantation of hAMSCs ameliorated ISO-induced development of cardiac injury in male Wistar rats. The findings of current study suggest that transplantation of hAMSCs confers cardioprotection by targeting ISO-induced mitochondriadependent (intrinsic) pathway of apoptosis.

\section{Acknowledgments}

Present work was funded by a research grant from Physiology Research Center in Iran University of Medical Sciences. Compliance with ethical standards.

\section{Conflict of Interests}

The authors declare that they have no competing interests.

\section{References}

1. Bonora M, Wieckowski MR, Sinclair DA, Kroemer G, Pinton P, Galluzzi LJNRC. Targeting mitochondria for cardiovascular disorders: therapeutic potential and obstacles Nat Rev Cardiol. 2018:1.

2. Souri F, Rakhshan K, Erfani S, Azizi Y, Maleki SN, Aboutaleb NJI. Natural lavender oil (Lavandula angustifolia) exerts cardioprotective effects against myocardial infarction by targeting inflammation and oxidative stress. Inflammopharmacology. 2018:1-9.

3. Rakhshan K, Azizi Y, Naderi N, Afousi AG, Aboutaleb $\mathrm{N}$ et al, ELABELA (ELA) peptide exerts Cardioprotection against myocardial infarction by targeting oxidative stress and the improvement of heart function. Int J Pept Res Ther. 2018:1-9.

4. Gorjipour F, Totonchi Z, Gholampour Dehaki M, Hosseini S, Tirgarfakheri K, Mehrabanian M, et al. Serum levels of interleukin-6, interleukin-8, interleukin-10, and tumor necrosis factor-alpha, renal function biochemical parameters and clinical outcomes in pediatric cardiopulmonary bypass surgery. Perfusion. 2019:267659119842470.

5. Talman V, Ruskoaho HJC, research t. Cardiac fibrosis in myocardial infarction-from repair and remodeling to regeneration. Cell Tissue Res. 2016;365(3):563-81

6. Lefer DJ, Granger DNJTAjom. Oxidative stress and cardiac disease. Am. J. Med. 2000;109(4):315-23.

7. Ghardashi AA, Gaeini A, Rakhshan K, Naderi N, Darbandi AA, Aboutaleb N, et al. Targeting necroptotic cell death pathway by highintensity interval training (HIIT) decreases development of postischemic adverse remodelling after myocardial ischemia/reperfusion injury. J Cell Commun. Signal 2018;13:255-267

8. Othman AI, Elkomy MM, El-Missiry M, Dardor MJEjop. Epigallocatechin-3-gallate prevents cardiac apoptosis by modulating the intrinsic apoptotic pathway in isoproterenol-induced myocardial infarction. Eur J Pharmacol. 2017;794:27-36.

9. Li A-Y, Wang J-J, Yang S-C, Zhao Y-S, Li J-R, Liu Y, et al. Protective role of Gentianella acuta on isoprenaline induced myocardial fibrosis in rats via inhibition of NF- $\kappa \mathrm{B}$ pathway. Biomed Pharmacother. 2019;110:733-41.

10. Bhutani S, Nachlas AL, Brown ME, Pete T, Johnson CT, Garcia AJ, et al. Evaluation of hydrogels presenting extracellular matrix-derived adhesion peptides and encapsulating cardiac progenitor cells for cardiac repair. ACS Biomater Sci Eng. 2017;4(1):200-10.

11. Amani H, Mostafavi E, Arzaghi H, Davaran S, Akbarzadeh A, Akhavan $\mathrm{O}$, et al. Three-dimensional graphene foams: synthesis, properties, biocompatibility, biodegradability, and applications in tissue engineering. ACS Biomater Sci Eng. 2018;5(1):193-214.

12. Saporito F, Baugh LM, Rossi S, Bonferoni MC, Perotti C, Sandri G, et al. In Situ Gelling Scaffolds Loaded with Platelet Growth Factors to Improve Cardiomyocyte Survival after Ischemia. ACS Biomater Sci Eng. 2018;5(1):329-338.

13. Bagno L, Hatzistergos KE, Balkan W, Hare JMJMT. Mesenchymal stem cell-based therapy for cardiovascular disease: progress and challenges. Mol Ther. 2018;26(7):1610-1623

14. Golpanian S, Wolf A, Hatzistergos KE, Hare JMJPR. Rebuilding the damaged heart: mesenchymal stem cells, cell-based therapy, and engineered heart tissue. Physiol Rev. 2016;96(3):1127-68.
15. Li L, Zhang Y, Li Y, Yu B, Xu Y, Zhao S, et al. Mesenchymal stem cell transplantation attenuates ciardiac fibrosis associated with isoproterenol-induced global heart failure. Transpl Int. 2008;21(12):1181-9.

16. Kadry MO, Abdel-Megeed RMJCt. Novel Mesenchymal Stem Cell Strategy in Alleviating Toll-Like Rreceptor-4, p53 and Nrf2 Signaling in Isoproterenol-Induced Myocardial Infarction in Rat Model. Cardiovasc Toxicol. 2018;18(3):232-41.

17. Gorjipour F, Hosseini-Gohari L, Alizadeh Ghavidel A, Hajimiresmaiel SJ, Naderi N, Darbrandi Azar A, et al. Mesenchymal stem cells from human amniotic membrane differentiate into cardiomyocytes and endothelial-like cells without improving cardiac function after surgical administration in rat model of chronic heart failure. J Cardiovasc Thorac Res. 2019;11(1):35-42.

18. Li D, Qu Y, Tao L, Liu H, Hu A, Gao F, et al. Inhibition of iNOS protects the aging heart against $\beta$-adrenergic receptor stimulationinduced cardiac dysfunction and myocardial ischemic injury. J Surg Res. 2006;131(1):64-72.

19. Verma VK, Malik S, Narayanan SP, Mutneja E, Sahu AK, Bhatia J, et al. Role of MAPK/NF- $\mathrm{B}$ pathway in cardioprotective effect of Morin in isoproterenol induced my/ocardial injury in rats. Mol Biol Rep. 2019:1-10.

20. Sagor M, Taher A, Tabassum N, Potol M, Alam MJOm, longevity c. Xanthine oxidase inhibitor, allopurinol, prevented oxidative stress, fibrosis, and myocardial damage in isoproterenol induced aged rats. Oxid Med Cell Longev. 2015;2015.

21. Pan J, Alimujiang M, Chen Q, Shi H, Luo XJJocb. Exosomes derived from miR-146a-modified adipose-derived stem cells attenuate acute myocardial infarction- induced myocardial damage via downregulation of early growth response factor 1 . J Cel. Biochem. 2019;120(3):4433-43

22. Amani H, Habibey R, Hajmiresmiail S, Latifi S, Pazoki-Toroudi H, Akhavan $\mathrm{O}$, et al. Antioxidant nanımaterials in advanced diagnoses and treatments of ischemia reperfiusion injuries. J Mater Chem B. 2017;5(48):9452-76.

23. Padmanabhan $M$, Prince PSMJLs. S-allylcysteine ameliorates isoproterenol-induced cardiac toxicity in rats by stabilizing cardiac mitochondrial and lysosomal enzymes. Life Sci. 2007;80(10):972-8.

24. Ebenezar KK, Sathish V, Devaki TJNr. Effect of arginine and lysine on mitochondrial function during isoproterenol induced myocardia infarction in rats. Nutr Res. 2003;23(9):1269-77.

25. Faezi M, Maleki SN, Aboutaleb N, Nikougoftar MJJocn. The membrane mesenchymal stem cell derived conditioned medium exerts neuroprotection against focal cerebral ischemia by targeting apoptosis. J Chem Neuroanat. 2018;94:21-31.

26. Saranya S, Baskaran R, Poormima P, Vijaya Padma VJJocb. Berbamine ameliorates isoproteremol-induced myocardial infarction by inhibiting mitochondrial dysfunction and apoptosis in rats. $\mathrm{J}$ Cell Biochem. 2018; 120(3): 3101-3113

27. Zhang Y, Köhler K, Xu J, Lu D, Braun T, Schlitt A, et al. Inhibition of p53 after acute myocardial infarction: reduction of apoptosis is counteracted by disturbed scar formation and cardiac rupture. J Mol Cell Cardiol. 2011;50(3):471-8. 